\title{
Sjögren's syndrome-trishna predominant amavata? A case report
}

\begin{abstract}
Sjögren's syndrome (SS) is one of the three most common autoimmune systemic diseases. The pathogenesis of SS is still unknown. SS often has an insidious onset, a variable course, and a wide variety of clinical manifestations, making the diagnosis difficult or delayed. SS can present either alone (Primary Sjögren's Syndrome - pSS) or in association of an underlying connective tissue disease, most commonly 'Rheumatoid Arthritis (RA)' or 'Systemic Lupus Erythematosus (SLE)' (Secondary Sjögren's Syndrome - sSS). Clinically the hallmarks of SS are kerato-conjunctivitis sicca (dry eyes) and xerostomia (dry mouth), or named sicca complex. SS occasionally coexists with other systemic autoimmune diseases, such that SLE and RA. Treatment goals of SS includes, palliative management of symptoms, preventing complications and immunosuppressive agents. Ayurvedic concept of SS is obscure and studies are lacking in this area. Things have become difficult for an Ayurvedic physician to evaluate and manage the cases of SS due to lack of literature. The present case report deals with a patient of sSS (associated with RA) came for Ayurvedic treatment. The diagnosis of 'Amavata' has been made and treated accordingly. 'Trishna' mentioned in 'Amavata' seems similar to 'xerostomia' or 'sicca' of Sjögren's syndrome. The diagnosis and line of treatment of 'Amavata' is suitable to manage the condition of 'Secondary Sjögren's Syndrome' (sSS) especially when it is associated with 'Rheumatoid Arthritis'. Ayurvedic treatment looks promising to manage Sjögren's syndrome and its complications.
\end{abstract}

Keywords: ayurveda, amavata, autoimmune disease, rheumatoid arthritis, sjögren's syndrome, systemic lupus erythematosus
Volume 12 Issue 5 - 2019

\author{
Kshama Gupta, Prasad Mamidi \\ Department of Kayachikitsa,Abhilashi University, India
}

Correspondence: Kshama Gupta, Professor, Department of Kayachikitsa, Abhilashi University, Mandi, Himachal Pradesh, India, Tel 7567222309,Email drkshamagupta@gmail.com

Received: August 18,2019 | Published: October 07, 2019
Abbreviations: SS, sjögren's syndrome; pSS, primary sjögren's syndrome; RA, rheumatoid arthritis; SLE, systemic lupus erythematosus; ILD, interstitial lung disease; ANA, antinuclear autoantibodies

\section{Introduction}

Sjögren's syndrome (SS) is one of the three most common autoimmune systemic diseases. The pathogenesis of SS is still unknown. SS often has an insidious onset, a variable course, and a wide variety of clinical manifestations, making the diagnosis difficult or delayed. ${ }^{1}$ SS can present either alone (Primary Sjögren's Syndrome - pSS) or in association of an underlying connective tissue disease, most commonly 'Rheumatoid Arthritis (RA)' or 'Systemic Lupus Erythematosus (SLE)' (Secondary Sjögren's Syndrome sSS). Clinically the hallmarks of SS are kerato-conjunctivitis sicca (dry eyes) and xerostomia (dry mouth), or named sicca complex. SS occasionally coexists with other systemic autoimmune diseases, such that SLE and RA. ${ }^{2}$ sSS consists of a sicca complex, which may be relatively mild and associated with one of the autoimmune diseases. ${ }^{3}$ The presence of multiple SS related comorbidities like anxiety, depression and fibromyalgia, may influence the severity of patient symptoms and further complicate the evaluation process. Presently there is no cure for SS exists. Treatment goals of SS includes, palliative management of symptoms, preventing complications and immunosuppressive agents. ${ }^{4}$
Ayurvedic concept of SS is obscure and studies are lacking in this area. Things have become difficult for an Ayurvedic physician to evaluate and manage the cases of SS due to lack of literature. The present case report deals with a patient of sSS came for Ayurvedic treatment. Written informed consent was obtained from the patient for the publication of this case report and accompanying images.

\section{Case description}

A 52year old female patient came to our care (30.05.2017) with the complaints of pain \& swelling at small (interphalangeal joints of both hands) (Lt>Rt) and large joints (elbow, wrist, ankle and knee joints) $(\mathrm{L} t>\mathrm{Rt}$ ), fever (aggravates especially during evenings, nights and in winters), dryness of eyes and mouth, indigestion, loss of appetite, difficulty swallowing, cough (dry in nature and aggravates especially after sunset and during winters), dyspnea, depression, fatigue and body pains. Initially (2007) the patient was diagnosed as having 'RA' and took steroids \& NSAID's (Non steroidal anti-inflammatory drugs). Later on (since 2014), she has developed 'Osteoporosis', 'Interstitial lung disease (ILD)', hypertension, dry eyes, xerostomia, depression and skin lesions (inflammatory, circumscribed, nodular lesions over buttocks). All these signs\& symptoms have started insidiously and they were gradually progressive.

The patient has been taking NSAID's, corticosteroids, antihypertensives, calcium supplementation and laxatives regularly. She has been getting mild relief with the above medicines but she is 
not satisfied with that relief. Patient was non smoker, non alcoholic and doesn't have positive family history of any autoimmune disease. Patient has not been taking any medication for allergy on regular basis. Patient has opted for Ayurvedic treatment and came to our care in search of better and sustained relief. At the time of examination, swelling (without erythema) and deformities were noted at interphalangeal joints of both hands $(\mathrm{Lt}>\mathrm{Rt})$ and at wrists (Figure 1). Swelling associated with tenderness is noticed at both elbow joints (Figures $2 \& 3$ ), ankle joints and knee joints. Patient had mild fever and restricted movements at the above said joints of the body at the time of examination. Dryness of conjunctiva and oral cavity found. Skin was also dry and nodular, inflammatory, localized, well circumscribed, tender skin lesions were found on both buttocks (Figure 4). Patient had difficulty in breathing and non-productive cough was observed. Patient had problems in swallowing of hard foods and she required frequent sips of water to swallow such type of foods. Dryness of skin, conjunctiva and mouth aggravates especially after sunset, during winters and cloudy weathers. While conversation it was noticed that, patient had pessimistic thoughts and depressed mood. Blood pressure was normal $(120 / 80 \mathrm{~mm}$ of $\mathrm{Hg}$ ) (patient has been taking anti hypertensive medication). There was no parotid gland swelling. Bilateral axillary lymph node enlargement has been found on palpation.

Reduced haemoglobin levels found with increased levels of CRP (C-reactive protein), ESR (Erythrocyte Sedimentation Rate) and RF (Rhuematoid factor) (IgM) titre. X-Ray of cervical spine was normal. HRCT (High Resolution Computed Tomography) scan of chest \& abdomen has revealed, 'ILD' and 'Mild hepatomegaly' (Table 1). ANA (Antinuclear auto-antibodies) anti-Ro/SSA titre found positive which substantiates the diagnosis of 'SS'. Schirmer's test and labial salivary gland biopsy were not done due to the patient's non acceptance for these investigations.

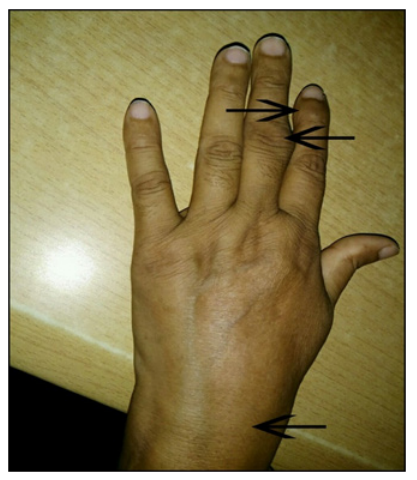

Figure I Inflammation of the left hand wrist and inter-phalangeal joints.

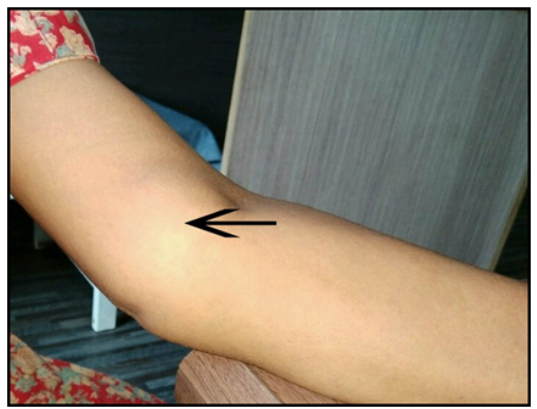

Figure 2 Swelling at medial side of the left elbow joint.

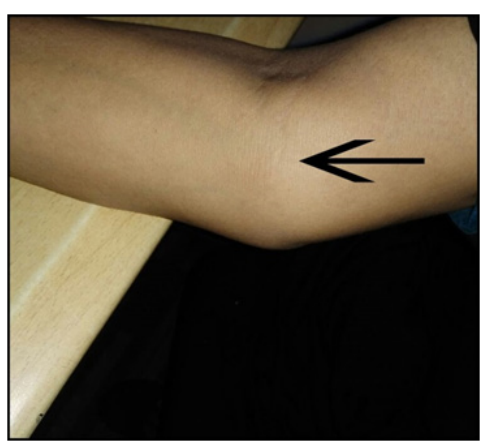

Figure 3 Swelling at medial side of right elbow joint.

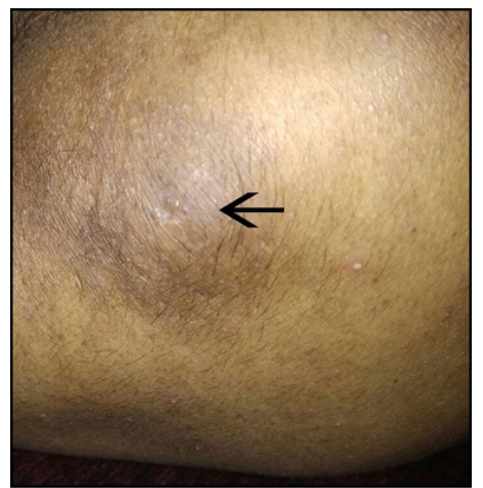

Figure 4 Skin lesions at gluteal region.

\section{Diagnosis, assessment \& treatment}

The existing US criteria for diagnosing 'SS' were for the first time integrated in the current classification by the major societies, ACR (American College of Rheumatology) and EULAR (European League Against Rheumatism). Patients experiencing sicca symptoms for at least 3months with positive 'Schirmer's test' or serological (Anti-Ro/ SSA) and histological examinations (labial salivary gland biopsy) strongly indicates the diagnosis of SS. Among these investigations, anti-Ro/SSA antibodies and an abnormal labial salivary gland biopsy have the highest specificity; consequently, they are the criteria with the highest values..$^{5}$ Although once considered the gold standard for diagnosis of SS, minor salivary gland biopsy of tissue taken from the patient's lip is not always necessary. ${ }^{1}$

In present case, the diagnosis of 'SS' was made based on the features like, dry eyes, dry mouth, skin manifestations, fatigue, involvement of lungs (ILD), lymphoproliferative conditions like hepatomegaly \& enlarged bilateral axillary lymph nodes, elevated ESR \& CRP and positive serological findings for 'RF' \& anti-Ro/SSA antibodies (Table 1). The diagnosis of 'sSS' was made by the presence of 'RA' features along with 'SS'. Ayurvedic diagnosis of 'Amavata' (inflammatory arthritis) has been made based on the features, 'angamarda' (generalized body pains), 'aruchi' (loss of appetite), 'trishna' (excessively thirsty), aalasyam' (fatigue), 'gauravam' (feeling of heaviness in body), 'jwara' (fever), 'shoonatanga' (swelling of body parts or joints), and 'apaaka' (indigestion). ${ }^{6}$ No assessments were carried out as the patient came for screening purpose initially and left against medical advice (LAMA) after few weeks of receiving treatment. The main objective of the treatment was, to manage 'saamavastha' and to manage 'trishna'. After achieving 'niramavastha', 'vata hara chikitsa' (vata dosha pacifying treatment) has been done. 
Table I Investigation reports

\begin{tabular}{|c|c|c|}
\hline Date & Name of the investigation & Report \\
\hline \multirow{9}{*}{30.05 .2017} & Hemoglobin & $10.2 \mathrm{gm} / \mathrm{dl}$ \\
\hline & TLC & $8400 \mathrm{cu} \mathrm{mm}$ \\
\hline & RBC count & 3.82million cells $/ \mathrm{mcL}$ \\
\hline & Platelet count & $3,48,000$ \\
\hline & CRP & $32 \mathrm{mg} / \mathrm{liter}$ \\
\hline & ESR & $22 \mathrm{~mm} / \mathrm{hr}$ \\
\hline & RF (Rheumatoid factor) (IgM) titre & $76.31 \mathrm{IU} / \mathrm{ml}$ \\
\hline & Serum calcium & $9.2 \mathrm{mg} / \mathrm{dl}$ \\
\hline & ASO (Anti Streptolysin O) test & $182 \mathrm{IU} / \mathrm{ml}$ \\
\hline 30.05 .2017 & X-Ray of cervical spine & Normal \\
\hline 09.06 .2017 & HRCT Chest & $\begin{array}{l}\text { Mild inter and intra lobular septal thickening seen in the lower lobes of both } \\
\text { lungs mainly in the sub pleural location. } \\
\text { Small nodule of around } 4 \mathrm{~mm} \text { in the right upper lobe. } \\
\text { Mild traction bronchiectasis seen in left lingular lobe. } \\
\text { Enlarged non necrotic bilateral axillary lymph nodes seen. The largest node in } \\
\text { the right axilla measures around } 25 \times 22 \mathrm{~mm} \text {. }\end{array}$ \\
\hline 09.06 .2017 & HRCT Abdomen & $\begin{array}{l}\text { Mild hepatomegaly (around } 16 \mathrm{~cm} \text { ) } \\
\text { Physiologically distended gall bladder }\end{array}$ \\
\hline 09.06 .2017 & $\begin{array}{l}\text { ANA (Antinuclear auto-antibodies) } \\
\text { (anti-Ro/SSA titre) }\end{array}$ & Positive \\
\hline
\end{tabular}

\section{Discussion}

SS mainly affects women in the fourth and fifth decade with female to male ratio is $9: 1 .^{2} \mathrm{SS}$ has a wide variety of presentations, ranging from the local involvement of exocrine glands with keratoconjunctivitis sicca (KCS) and xerostomia to the systemic, extra glandular involvement of multiple organs. Sicca symptoms are the most common manifestation of SS. Fatigue also markedly worsens the quality of life of SS patient's. Patients with KCS complain about foreign-body sensation, burning or soreness of the eyes and increased sensitivity to light. Marked xerostomia presents clinically as, difficulties when talking and while chewing or eating dry food. The most common extra glandular manifestations are arthralgia and non-erosive polyarthritis. 'sSS' appears in the setting of another autoimmune disease, particularly SLE (15-36\%), RA (20-32\%), and limited or progressive systemic sclerosis (11-24\%). ${ }^{5}$ Although there is no definitive evidence to support screening guidelines for lymphoproliferative diseases in SS patients, the following features should raise the physician's index of suspicion like, enlarged parotid glands, regional or generalized lymphadenopathy, hepato-splenomegaly, pulmonary infiltrates, vasculitis, and hypergammaglobulinemia. ${ }^{1}$ Symptoms related to pulmonary involvement in SS patients vary from dry cough to dyspnea from ILD. Both rheumatoid factor and antinuclear antibodies are frequently found in both primary and secondary SS. ${ }^{3}$

Presently, no cure or remittive agent for SS exists. Treatment goals remain symptom palliation, prevention of complications and immunosuppressive therapy. ${ }^{4}$ Disease-modifying therapy is reserved for SS patients with systemic involvement, but there is limited evidence for its efficacy. Because of the complexity of this disease, some of its clinical manifestations may require interdisciplinary management. ${ }^{5}$ Patients with sSS have old age \& long duration of the disease compared to $\mathrm{pSS}^{2}$ The present case after receiving ten years of treatment with various medicines like corticosteroids, NSAID's, multi vitamins and laxatives etc patient opted for Ayurvedic treatment to arrest progression of the disease, to prevent complications (like ILD, hepatomegaly etc in present case) and to get satisfactory and sustained relief. The patient was diagnosed as having 'sSS' according to modern medicine and 'Amavata' according to Ayurveda.

'Shushkakshipaka' (inflammatory eye disease associated with dryness) is mentioned in the classical literature of Ayurveda under 'Sarvagata Netraroga' (diseases affecting all parts of the eye). KCS or dry eye syndrome is having similarity with 'Shushkakshipaka'? Amavata is a disease in which vitiation of 'Vata dosha' and accumulation of 'Ama' (maldigested, non-homogeneous product) takes place in joints, and leads to production of pain, stiffness, swelling, tenderness, etc., in the related joins. The features of Amavata are much identical to RA, an autoimmune disorder which causes chronic inflammatory and symmetrical polyarthritis. ${ }^{8}$ If the condition is allowed to progress the pain may begin to migrate from one joint to another joint, with an intense sting or burning sensation. ' 'Vatarakta' is a disease caused by vitiated 'Vata' and 'Rakta', which can provide some insights to understand the concept of autoimmunity according to Ayurveda. ${ }^{9}$

In present case, clinical features like generalized body pains, loss of appetite, indigestion, constipation, dry mouth or requirement of frequent sips of water while eating solid foods, fatigue, feeling of heaviness in body, fever, indigestion and arthralgia etc indicates the diagnosis of 'Amavata' (which is characterized by anagamarda, aruchi, trishna, aalasyam, gauravam, jwara, apaaka, shoonataanga etc signs and symptoms). Among them 'Trishna' (feeling of excessive thirstiness) was predominant and it is similar to 'Xerostomia' or dry mouth of SS. Oral symptoms in SS include difficulty speaking, eating, or swallowing, and frequent sips of water while eating food 
or in general. ${ }^{1}$ This xerostomia or sicca features of SS clearly shows similarity with 'trishna' of 'Amavata'. In present case, the whole clinical picture (SS with RA or sSS) has shown similarity with 'Amavata' (with the predominance of 'trishna').

After making the diagnosis of 'Amavata', the patient has received 'ama paachana' (drugs or treatment which rectifies ama dosha) and 'trishna prashamana' (drugs which controls excessive thirstiness) drugs. Before starting Ayurvedic medicines, all modern medicines like steroids, NSAID's, laxatives, anti-hypertensives and calcium supplementation etc were stopped. 'Bala jeerakadi kashayam' or 'Nayopayam kashayam' were used to manage dry cough and dyspnea. 'Ashtavargam kashayam' or 'Gandharvahastadi kashayam' were used to manage arthralgia symptoms and also for 'ama pachana' purpose. 'Shunti siddha jala pana' (luke warm water fortified with dry ginger) has been given to the patient to manage 'trishna'. After taking the above medication for two weeks patient got relief in dry cough, dyspnea, fever, body pains, constipation, joint pains and swelling and loss of appetite. After achieving 'niramavastha', 'panchatikta ghritam' has been prescribed as 'shamana snehapana' ( $10 \mathrm{ml}$ of ghee twice a day before meals with hot water). Patient got good improvement in arthralgia features, dry eyes and dry mouth, cough, dyspnea, fatigue and also in skin lesions after taking 'panchatikta ghrita' for one month. Then patient suddenly stopped treatment and went for pilgrimage (became LAMA). Treatment was stopped by the patient abruptly against the medical advice and there was no follow up or communication later on.

No adverse effects were reported with the above Ayurvedic drugs by the patient during treatment period. The patient got clinically meaningful improvement with Ayurvedic medicines when they were administered especially in KCS and in arthralgia features. Patient has discontinued the treatment. Ayurvedic treatment seems to be promising in the management of sSS (associated with RA) without causing any adverse effects. The diagnosis and management protocol of 'Amavata' is suitable for the patients of sSS (especially when SS is associated with RA).

\section{Limitations of the present study}

In present case minor salivary gland biopsy has been not done due to reluctance of the patient for this invasive procedure. The diagnosis is solely made on history, clinical and serological findings. Assessment was not done (as the patient became LAMA) and no objective parameters were used in the present study. Present study findings can't be generalized and further experimental and / or clinical studies with large sample are required to substantiate the present study observations.

\section{Conclusion}

'Trishna' mentioned in 'Amavata' seems similar to 'xerostomia' or 'sicca' of Sjögren's syndrome. The diagnosis and line of treatment of 'Amavata' is suitable to manage the condition of 'Secondary Sjögren's Syndrome' (sSS) especially when it is associated with 'Rheumatoid Arthritis'. Ayurvedic treatment looks promising to manage Sjögren's syndrome and its complications.

\section{Acknowledgments}

None.

\section{Conflicts of interest}

The authors declare there are no conflicts of interest related to the article.

\section{References}

1. Kruszka P, O’Brian RJ. Diagnosis and management of Sjögren syndrome. Am Fam Physician. 2009;79 (6):465- 472

2. Abdulkareem FA, AL-Aswad FD, Alasami MH. The incidence of secondary sjogren syndrome in relation to disease activity \& disease duration of rheumatoid arthritis patients. International Journal of Science and Research. 2017;6(5):97-100.

3. Mahendranath KM. Sjogren's Syndrome-Diagnosis and Management. JAPI. 2006;54(N):54-57.

4. Vivino FB, Carsons SE, Foulks G, et al. New treatment guidelines for Sjögren's disease. Rheum Dis Clin North Am. 2016;42(3):531-551.

5. Stefanski AL, Tomiak C, Pleyer U, et al. The diagnosis and treatment of Sjögren's syndrome. Dtsch Arztebl Int. 2017;114(20):354-361.

6. Sharma UK, Kumawat VB, Sharma P, Bhaskar R, Sharma SK. Clinical evaluation of virechana therapy in Amavata with special Reference to Rheumatoid Arthritis. AYUSHDHARA. 2016;3(5):822-826.

7. Dhiman KS. Shushkakshipaka (dry eye syndrome): A case study. Int $J$ Ayurveda Res. 2011;2(1):53-55.

8. Gupta SK, Thakar AB, Dudhamal TS, et al. Management of Amavata (rheumatoid arthritis) with diet and Virechanakarma. $A Y U$. $2015 ; 36(4): 413-415$.

9. Ramteke R. Management of Rheumatoid Arthritis through Ayurveda. $J$ Tradi Med Clin Natur. 2016;5:189. 